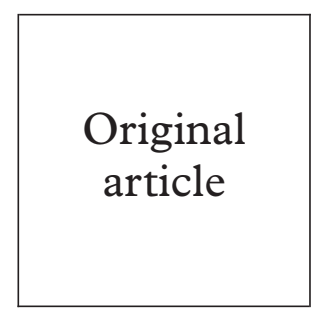

Original article

Laboratory Sciences Division, ICDDR,B, Dhaka, Bangladesh J Bogaerts M Rahman

S Nahar

Bangladesh Women's

Health Coalition, Dhaka, Bangladesh J Ahmed

N Akhter

$\mathrm{N}$ Begum

Laboratory of Microbiology,

University of Leuven, Belgium

M Van Ranst

J Verhaegen

Correspondence to: J Bogaerts, Laboratory Sciences Division,

ICDDR,B, GPO Box 128, Dhaka-1000, Bangladesh bogaerts@icddrb.org

Accepted for publication 12 October 2000

\title{
Sexually transmitted infections among married women in Dhaka, Bangladesh: unexpected high prevalence of herpes simplex type 2 infection
}

\author{
J Bogaerts, J Ahmed, N Akhter, N Begum, M Rahman, S Nahar, M Van Ranst, \\ J Verhaegen
}

Objectives: To document the prevalence of reproductive tract infections (RTI) and sexually transmitted infections (STI) among women attending a basic healthcare clinic in Dhaka, Bangladesh, to identify risk factors associated with the diseases and to estimate the incidence of syphilis, hepatitis C (HCV), hepatitis B (HBV), and herpes simplex type 2 (HSV-2) infection.

Methods: A cross sectional sample of 2335 consecutive women was examined during 1996-8. Women were interviewed about risk factors for RTI/STI and tested for Neisseria gonorrhoeae, Chlamydia trachomatis, Trichomonas vaginalis, Treponema pallidum, HIV, HCV, HBV, HSV-1 and HSV-2 infection as well as vaginal candidosis and bacterial vaginosis. Women with antibodies to $T$ pallidum were retested at regular intervals. One year after ending the study seroconversion for syphilis, HBV, HCV, and HSV-2 infection was detected among women initially negative for the respective diseases.

Results: The overall prevalence rate of $N$ gonorrhoeae, $C$ trachomatis, $T$ vaginalis, and $T$ pallidum infection was $0.5 \%, 1.9 \%, 2.0 \%$, and $2.9 \%$ respectively. Overall, $35 \%$ of the women had antibodies to hepatitis $\mathrm{B}$ core antigen, $0.9 \%$ had HCV, and $12 \% \mathrm{HSV}-2$ infection. Risk factors for gonorrhoea/C trachomatis infection were a husband not living at home or suspected of being unfaithful. HSV-2 infection was associated with the same risk factors and with a polygamous marriage. The prevalence of HSV-2 infection among women "at risk" was $23 \%$. HIV infection was not diagnosed. Repeated serological examination indicated that only $32 \%$ of women with serological evidence of syphilis had active disease. The seroincidences of HBV, HCV, and HSV-2 were $0.03,0.007$, and 0.009 per person year. Seroconversion for syphilis was not observed. (Sex Transm Inf 2001;77:114-119)

Keywords: sexually transmitted infections; genital herpes; Bangladesh

\section{Introduction}

Little information exists on the prevalence of reproductive tract infections/sexually transmitted infections (RTI/STI) in Bangladesh. During 1985-6, the prevalence of gonorrhoea, Chlamydia trachomatis, and Trichomonas vaginalis infection was $0.4 \%, 2 \%$, and $5 \%$ respectively among symptomatic married women in a rural area. ${ }^{1}$ Ten years later identical rates were found in a similar population in the same geographic area. ${ }^{2}$ The present study was undertaken to eliminate the lack of information on RTI/STI among urban women.

\section{Methods}

A cross sectional sample of 2335 consecutive new female clients, attending a basic healthcare clinic in Mirpur, Dhaka, from July 1996 till April 1998, was included in the study. Recruitment was done by selecting women after registration, in order of arrival at the clinic, and before any interview or physical examination was performed. Women were grouped according to the reason of their visit. Consenting women were interviewed in a private room by a social worker to obtain information about sociodemographic characteristics (age, religion, civil status, education, income, current family planning method, occupation of the husband and woman), risk factors of RTI/STI related to the husband (current signs of STI, number of marriages and legal wives, extramarital relationships) and to the women themselves (number of marriages, extramarital relationships, sanitary protection, vaginal douching after intercourse, symptoms of RTI/ STI in the past, current symptoms of vaginal discharge, and medical history). When it became clear that the vast majority of women attending the clinic for other reasons than vaginal discharge declared themselves to suffer from vaginal discharge the question about the presence of discharge was replaced by one asking whether and since when a change in vaginal discharge was observed. After the interview a clinical examination of the external genitals and a bimanual pelvic examination were performed. Clinical examination was done by a nurse under supervision of a trained female doctor.

Culture of $N$ gonorrhoeae from the endocervix was performed according to recommended methods; detection of $C$ trachomatis was done by an ELISA technique with blocking assay. The diagnosis of bacterial vaginosis (BV) was made on Gram staining according to the Nugent criteria. ${ }^{3}$ Vaginal candidosis was diagnosed by microscopic fresh examination and Gram staining of the vaginal fluid, $T$ vaginalis infection by fresh examination only.

Blood was drawn for the detection of antibodies and/or markers of Treponema pallidum, hepatitis B virus (HBV), and hepatitis C 
virus (HCV) as well as herpes simplex virus (HSV) type 1 and HSV-2 infection. For the diagnosis of syphilis, the rapid plasma reagin test (RPR) was performed and the RPR titre was determined (RPR-nosticon II, Organon Teknika, Boxtel, Netherlands). Antibodies to $T$ pallidum were detected using a microhaemagglutination test (TPHA-nosticon, Organon Teknika) or a passive particle agglutination test (Serodia TPPA, Fujirebio, Tokyo, Japan). The TPHA test was performed on 1189 and the TPPA test on 1142 women; four women did not give a blood sample. Active syphilis was considered when the RPR test was reactive with a positive TPHA/TPPA test, any reactive RPR titre being considered significant. $T$ pallidum IgG and IgM antibodies were detected by an ELISA technique among patients with a reactive RPR and/or positive TPHA/TPPA test (Treponema pallidum IgM EIA, Treponema pallidum IgG Comfort EIA Meddens Diagnostics, Brummen, Netherlands). Samples positive for IgM antibodies were checked for the rheumatoid arthritis (RA) factor to identify false positive IgM $T$ pallidum tests, using a particle agglutination test kit (Serodia RA, Fujirebio, Tokyo, Japan). An ELISA technique was used for the detection of antibodies and/or markers of HBV, HCV, HSV-1, and HSV-2 infection. Women were first tested for hepatitis B core antibodies (anti-HBcAg, total antibody) (Hepanostika anti-HBc, Organon Teknika). The hepatitis B surface antigen ( $\mathrm{HBsAg}$ ) was subsequently detected among anti-HBcAg positive women (Hepanostika HBsAg Uniform II, followed by HBsAg Uniform II, Confirmatory, Organon Teknika). HCV infection was detected using synthetic peptides from the non-structural NS2, NS3, and NS4 regions to detect serum antibodies (UBI HCV EIA 4.0, Beijing, People's Republic of China). Reactive samples were again retested with a second generation ELISA test (HCV EIA test, Abbott Laboratories, North Chicago, IL, USA) as well as with a line immunoassay (Inno-LIA HCV $\mathrm{Ab}$ III, Innogenetics, Zwijndrecht, Belgium). HSV-1 and HSV-2 infection were diagnosed by detecting specific IgG antibodies to glycoprotein $\mathrm{G}$ of $\mathrm{HSV}-1$ and $\mathrm{HSV}-2$ (HSV-1 specific IgG and HSV-2 specific IgG ELISA, Gull Laboratories Inc, Salt Lake City, UT, USA). Since HSV-1 antibodies were diagnosed among the overwhelming majority of women this test was performed for 183 patients. Detection of HSV-2 antibodies was done after the end of the study. HSV-1 and HSV-2 antibodies were detected among 79 prepubertal children, 1-12 years old, who submitted a blood sample to the clinical laboratory of ICDDR,B for various reasons. HIV antibodies were detected by an ELISA technique (Vironostika HIV Uni-Form II plus O, Organon Teknika). Reactive samples were retested in a line immunoassay (Liatek HIV III, Organon Teknika). Testing was unlinked and anonymous.

TREATMENT AND FOLLOW UP

Women were treated according to a World Health Organization recommended flow chart for vaginal/cervical discharge. ${ }^{4}$ Women with a reactive syphilis serology were treated by a weekly intramuscular injection of 2.4 million units of benzathine penicillin for 3 consecutive weeks. ${ }^{5}$ Patients with syphilis were requested to return after $3,6,12,18$, and 24 months for serological follow up. When they failed to return a social worker visited the home and encouraged them to visit the clinic. A significant rise or decline of the RPR titre was diagnosed when a fourfold change in titre, equivalent to a change of two dilutions, was observed at follow up. During April-May 1999, 1 year after inclusion of the last patient, 1146 randomly selected homes were visited to document seroconversion for syphilis, $\mathrm{HBV}, \mathrm{HCV}$, and HSV-2 infection among women initially negative for the diseases. Seroconversion for HSV-2 was detected by simultaneous testing of the first and last serum specimen using the same test kit.

STATISTICAL ANALYSIS

Data were analysed using the EPI-INFO software, Version 6, WHO Geneva, Switzerland. The Yates's corrected $\chi^{2}$ test (when appropriate) was applied to assess differences in proportions for statistical significance. The odds ratio (OR) was used for measuring associations. Logistic regression was performed with the sPss software package, version 7.5 , using indicators significantly associated with the respective diseases in bivariate analysis. All variables were simultaneously entered in the model. The seroincidence of syphilis, HBV, HCV, and HSV-2 infection was estimated by the ratio of the number of seroconversions divided by the number of person years of follow up.

\section{Results}

CLIENTS

Ninety six per cent $(2335 / 2439)$ of the women approached agreed to participate in the study. Of the participating women only $2.4 \%(n=55)$ was divorced/separated, $0.6 \% \quad(n=15)$ widowed, and $1.2 \%(n=28)$ unmarried. Ninety eight per cent were Muslim. The following groups were constituted: antenatal care (AC, $\mathrm{n}=308$ ), family planning ( $\mathrm{FP}, \mathrm{n}=688)$, pregnancy interruption (PI, $n=698$ ), expanded programme on immunisation (EPI, $n=185$ ), and vaginal discharge $(\mathrm{VD}, \mathrm{n}=456)$. The 28 unmarried women were excluded from speculum examination and could not be interviewed about sexual behaviour or use of contraceptive methods. Except for serological tests they did not have laboratory examination. Load shedding and erratic supply of laboratory reagents prevented the diagnosis of $T$ vaginalis, gonorrhoea, and chlamydia infection among 87,191 , and 336 women, respectively. A total of 1879 $(81.4 \%)$ of 2307 women were tested for all cervical and vaginal pathogens.

SOCIODEMOGRAPHIC DATA

The mean age of the 1879 women was 25.6 (SD 6.3) years. Widows (mean age 32.7 (10.2) years) were significantly older than married women (mean age 25.8 (6.2) years), who were older than separated/divorced women (mean 
Table 1 Clinical and laboratory findings among women attending a basic healthcare clinic in Dhaka

\begin{tabular}{|c|c|c|c|c|c|c|}
\hline & \multicolumn{6}{|c|}{ Mean age (SD) and percentage of women with corresponding characteristics } \\
\hline & \multicolumn{6}{|l|}{ Client groups $\neq$} \\
\hline & $A C(n=243)$ & $E P I(n=144)$ & $F P(n=555)$ & $P I(n=592)$ & $V D(n=345)$ & $\begin{array}{l}\text { Overall } \\
(n=1879)\end{array}$ \\
\hline Mean age (years) & $21.9(4.4)$ & $23(4.9)$ & $26.8(6.5)$ & $25.6(6.0)$ & $27.9(6.5)$ & $26(6.3)$ \\
\hline Polygamous marriage & 2.5 & 5.6 & 9.3 & 10 & 8.6 & 8.3 \\
\hline $\begin{array}{l}\text { Symptoms } \\
\text { Change in vaginal discharge }\end{array}$ & 25 & 22 & 31 & 30 & $75 \$$ & 36 \\
\hline $\begin{array}{l}\text { Speculum examination } \\
\text { Abnormal vaginal discharge } \\
\text { Cervical mucopus }\end{array}$ & $\begin{array}{l}13 \\
10\end{array}$ & $\begin{array}{l}13 \\
6\end{array}$ & $\begin{array}{l}10 \\
12\end{array}$ & $\begin{array}{l}14 \\
14\end{array}$ & $\begin{array}{l}33 \$ \\
33 \S\end{array}$ & $\begin{array}{l}15 \\
15\end{array}$ \\
\hline \multicolumn{7}{|l|}{ Laboratory findings } \\
\hline$N$ gonorrhoeae & 0.8 & - & 0.5 & 0.5 & 0.6 & 0.5 \\
\hline$C$ trachomatis & 2.5 & 2.1 & 1.3 & 2.0 & 2.0 & 1.9 \\
\hline Any cervical pathogen & 2.9 & 2.1 & 1.6 & 2.4 & 2.3 & 2.2 \\
\hline \multicolumn{7}{|l|}{ Vaginal pathogens } \\
\hline T vaginalis & 1.2 & 1.4 & 1.6 & 1.7 & 3.8 & 2.0 \\
\hline Candida $\mathrm{sp} \dagger$ & 25 & 17 & 24 & 24 & 17 & 21 \\
\hline Bacterial vaginosist & 41 & 28 & 28 & 24 & 30 & 29 \\
\hline BVTV† & 41 & 33 & 29 & 31 & 32 & 32 \\
\hline Any vaginal pathogen $\dagger$ & 63 & 50 & 51 & 54 & 47 & 51 \\
\hline \multicolumn{7}{|l|}{ Antibodies to: } \\
\hline Tpallidum $\bullet$ & 2.1 & 2.8 & 3.4 & 2.7 & 3.2 & 2.9 \\
\hline $\mathrm{HBcAg}$ & 33.9 & 34.7 & 35.7 & 32.7 & 40.6 & 35.3 \\
\hline HSV-2 & 7.9 & 9.8 & 10.5 & 13.6 & 15.4 & 12.0 \\
\hline
\end{tabular}

$\ddagger$ Tested for all cervical and vaginal pathogens.

$\mathrm{AC}=$ antenatal care, $\mathrm{EPI}=$ expanded programme on immunization, $\mathrm{FP}=$ family planning, $\mathrm{PI}=$ pregnancy interruption, $\mathrm{VD}=$ vaginal discharge.

BVTV = bacterial vaginosis or $T$ vaginalis infection, $\mathrm{HBcAg}=$ hepatitis $\mathrm{B}$ core antigen, $\mathrm{HSV}-2=$ herpes simplex virus type 2.

TOnly currently married women.

^No information for 38 women.

+Women with abnormal vaginal discharge.

$\$ \mathrm{p}<0.001$ between vaginal discharge and other groups.

$\bullet$ RPR reactive and TPHA positive.

age 22.6 (5.0) years). The mean age of the unmarried women was 16.9 (2.64) years. The mean income per household of married women was $5000 \mathrm{Taka} / \mathrm{month}$ (\$US110). The mean income of the widowed/divorced/separated group was $1770 \mathrm{Taka} / \mathrm{month}$ (\$39). The literacy rate was $59 \%$ : $32 \%$ had finished the primary school, $19 \%$ the lower, and $5 \%$ the higher secondary school, and 3\% had attended graduate training. Of all married women, 281 $(12.6 \%)$ had the husband living outside Dhaka or spending the night outside the house. Twenty three per cent of the married women was employed as factory workers, domestic helpers, or in other occupations versus $74 \%$ of the women in the other group. Ninety seven per cent of the husbands were in formal employment. The most frequent occupations were domestic helpers, shop keepers, factory workers, drivers, and rickshaw pullers. Overall, 17\% of the women had a history of stillbirth or spontaneous abortion and $36 \%$ had at least one induced abortion in the past. After exclusion of women attending the AC and EPI services as well as unmarried and widowed/divorced/ separated women, who could not be interviewed about the issue, the overall use of contraceptives was $32 \% \quad(553 / 1746)$. Oral contraceptives were currently used by $10 \%$, condoms by $9 \%$, injectable contraceptives by $8 \%$, an intrauterine device (IUD) by $3 \%$, and other methods by $2 \%$ of the women. The PI group had the lowest rate of contraceptive use ( $5 \%$ or $29 / 675)$. Ninety per cent of the women used rags or self made towels as menstrual protection, only three women used intravaginal tampons, whereas the remaining $10 \%$ did not use any protection. Eight per cent of the married women were living in a polygamous marriage whereas $6 \%$ of the women and $21 \%$ of the husbands had been married previously. Like unmarried women, widowed and divorced/separated women could not be interviewed about current sexual relationships. Five $(0.2 \%)$ married women admitted an extramarital partner. Sixty two per cent of the women had a history of vaginal discharge and 26\% performed vaginal douching after sexual intercourse. Eighteen per cent $(411 / 2237)$ of all married women reported urethral discharge, genital ulcers, or warts in the husband whereas $5 \%$ suspected them of being unfaithful.

SYMPTOMS AND SIGNS OF RTI/STI, LABORATORY FINDINGS

Symptoms, signs, and laboratory findings among women who were tested for all cervical and vaginal pathogens are represented in table 1. Overall, $3.0 \%(71 / 2331)$ of tested women had a reactive RPR with a positive TPHA/ TPPA and were considered as having syphilis, $0.5 \%(11 / 2116)$ gonorrhoea, $1.6 \%$ (38/1971) $C$ trachomatis, $1.8 \%(40 / 2220) T$ vaginalis, and $11.7 \%$ (272/2315) HSV-2 infection. Six $(0.3 \%)$ women combined a positive TPHA/ TPPA with a negative RPR whereas $24(1 \%)$ had a reactive RPR test with negative TPHA/ TPPA. Seventy five per cent (53/71) of the patients with syphilis showed a RPR titre of $<1$ :8. Seven $(9.9 \%)$ syphilis patients had $\operatorname{IgM}$ antibodies to T pallidum: $\operatorname{six}(33.3 \%)$ of 18 with RPR titre $\geqslant 1: 8 v 1(1.9 \%)$ of 53 with RPR titre $<1: 8$. At initial diagnosis, two $\operatorname{IgM}$ positive women had a borderline reactive RA test (titre, 1:40). Antibodies to $\mathrm{HBcAg}$ were observed among $35.2 \%(820 / 2328)$ women of whom 62 
Table 2 Indicators of sexually transmitted infections among married women in Dhaka

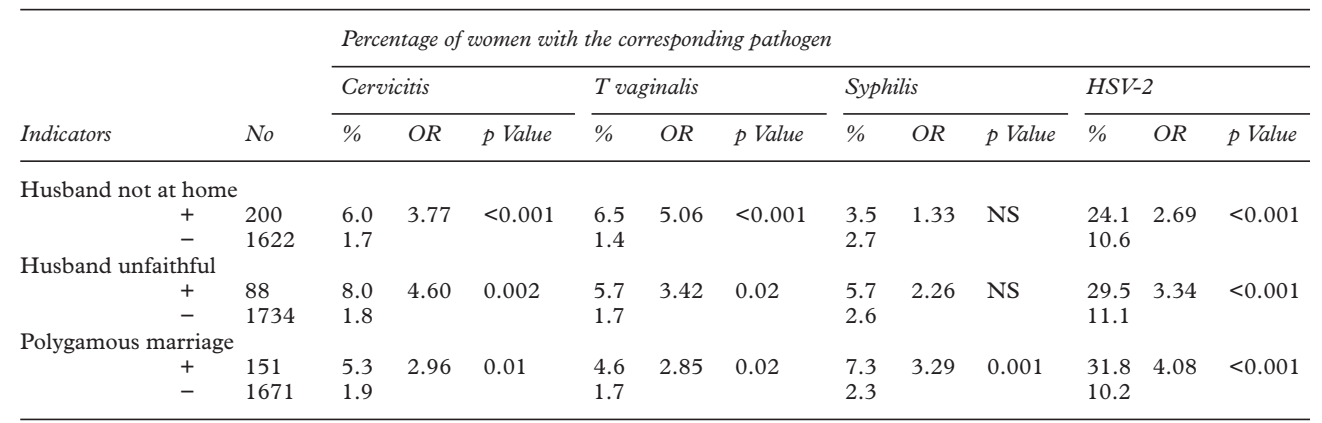

Cervicitis $=$ gonococcal or chlamydia cervicitis; HSV-2 $=$ herpes simplex virus type 2 infection; NS = not significant.

(7.6\%) were HBsAg positive. At initial screening $4.9 \%(114 / 2328)$ women tested positive for HCV antibodies. However, only 20 were confirmed in the second ELISA test or in the line immunoassay, indicating a $0.9 \%$ prevalence of $\mathrm{HCV}$ infection. Only two women showed a positive HIV screening test, not confirmed in the line immunoassay.

Since $97 \%(177 / 183)$ of initially tested women showed HSV-1 antibodies further screening was abandoned. Of the 79 prepubertal children, $46 \%$ and $2.5 \%$ had antibodies to HSV-1 and HSV-2 respectively. None of the 28 unmarried women had syphilis or $\mathrm{HCV}$ antibodies, five $(17.8 \%)$ showed antibodies to $\mathrm{HBcAg}$, one had antibodies to HSV-2. The prevalence of vaginal and cervical pathogens among women with and without signs of abnormal vaginal discharge has been published earlier. $^{6}$

RISK FACTORS OF STI

In bivariate analysis no association was found between any of the STI on one hand and family income, signs of RTI/STI in the partner, vaginal douching after sexual intercourse, or the use of sanitary protection or contraceptives. Bacterial vaginosis was observed among $41 \%$ (23/56) IUD users $v 13 \%(64 / 487)$ of users of other methods (OR, 4.61; $<<0.001)$ and $23 \%$ $(273 / 1193)$ of non-contraceptive users (OR, 2.35; $\mathrm{p}=0.003)$. Among women $<30$ years old, a history of fetal death (stillbirth or spontaneous abortion) was significantly associated with syphilis $(31 \%$ v $12 \%$; OR $3.18 ; \mathrm{p}=0.007)$. Common risk factors for STI are represented in table 2. After logistic regression gonococcal/ chlamydia cervicitis remained no longer associated with a polygamous marriage. $T$ vaginalis infection was only associated with a husband not at home and with abnormal vaginal discharge on clinical examination. Syphilis was no longer associated with a polygamous marriage but remained associated with increasing age, a history of fetal death, and lack of formal education. HSV-2 infection remained associated with age, syphilis, the husband not at home, unfaithful husband, and a polygamous marriage. Table 3 represents the age related prevalence of syphilis and HSV-2 infection. Antibodies to HBcAg were not associated with any STI or marker of STI and fluctuated between $30-35 \%$ among all age groups. Risk factors for new HBV infections were not identified.
FOLLOW UP OF SYPHILIS PATIENTS

Fifty two per cent (37/71) of syphilis patients returned for follow up. Of these, 12 had a single follow up visit. The mean delay between the first and second visit was 450 days (range 152979 days); no women were treated for syphilis before the second visit. A significant decrease and increase of the RPR titre was observed in two women respectively, two other women developed a non-reactive RPR, whereas eight others did not show any change in RPR titre. Twenty five women had repeated examinations, the mean duration of follow up being 497 days (range 147-945 days). All were treated after the first visit, as were 24 of 25 husbands. Only $10(40 \%)$ women showed a significant decrease of the RPR titre, four (16\%) became RPR negative and $11(44 \%)$ others remained unchanged. Five of seven $T$ pallidum IgM positive women had repeated examinations and showed a significant decrease in the RPR titre. Two of them remained IgM positive at 3 and 12 months after treatment. Considering a significant rise or decline of the RPR titre as a proof of active disease, only $32 \%(12 / 37)$ of women who had a follow up serum specimen had active syphilis. The mean age of these women (29.2 (SD 9.0) years) was comparable with the age of women who didn't show any titre change $(\mathrm{p}=0.72)$.

INCIDENCE OF SYPHILIS, HBV, HCV, AND HSV-2 INFECTION

Only $280(24.4 \%)$ of the 1146 randomly selected women could be contacted during the home visits in April to May 1999. Loss to follow up was due to the high mobility of the slum population, lack of accuracy in addresses, and the relatively high proportion of women in

Table 3 Age related prevalence of syphilis and HSV-2 infection among married women in Dhaka

\begin{tabular}{lllll}
\hline & & \multicolumn{2}{l}{$\%$ women with: } \\
\cline { 3 - 5 } & & \multicolumn{3}{c}{$\begin{array}{l}\text { HSV-2 infection } \\
\text { Risk factorst }\end{array}$} \\
\cline { 3 - 5 } Age (years) & No & Syphilis & + & - \\
\hline$<20$ & 281 & 1.4 & 10.0 & 3.3 \\
$20-24$ & 552 & 1.6 & 18.9 & 7.2 \\
$25-29$ & 478 & 2.5 & 24.5 & 12.6 \\
$30-34$ & 289 & 4.8 & 31.8 & 11.3 \\
$35-39$ & 172 & 6.4 & 27.5 & 15.4 \\
M & 50 & 0 & 40.0 & 8.9 \\
Overall & & 2.9 & 23.4 & 9.3 \\
\hline
\end{tabular}

HSV-2 = herpes simplex virus type 2 .

†Husband not at home, unfaithful, polygamous marriage. 
formal employment. Because of the significant association between syphilis and HSV-2 infection only women negative for both diseases at the initial visit were considered for calculating the seroincidence. Of the 231 returning women, 186 were negative for both syphilis and HSV-2, 148 for HBV, and 226 for HCV antibodies. The duration of follow up between the first and last blood sample was 349,272 , and 416 person years for the respective groups. No seroconversion was observed for syphilis, two women seroconverted for HCV (incidence 0.007 per person year), three for HSV-2 (incidence, 0.009 per person year), and 11 for HBV infection (incidence, 0.03 per person year).

\section{Discussion}

$N$ gonorrhoeae, $C$ trachomatis, and $T$ vaginalis infections constituted only a minority of the RTI among married women attending a basic healthcare clinic in Dhaka. The prevalence of those infections, except $T$ vaginalis infection, was strictly comparable among the different groups. The relatively low prevalence contrasts with findings from women in sub-Saharan Africa or central America. ${ }^{7-11}$

It is generally believed that the vast majority of women in Bangladesh do not have sexual relationships before or outside the marriage, in contrast with men. ${ }^{12}$ However, during a recent survey, about $50 \%$ of men and $25 \%$ of women were found to have experienced premarital sexual relations. Seven per cent of married men declared having had contact with a sex worker after marriage. Many of the sexual contacts with sex workers were reported to occur only once but a subgroup of married men having frequent contacts was recognised. Less than $10 \%$ of men used condoms during contact with sex workers. ${ }^{14}$ An ongoing study among street based sex workers showed that $72 \%$ were infected with HSV-2, whereas 35\% had syphilis and $25 \%$ gonorrhoea (unpublished data). In view of these findings and presuming that most married women suffering from an STI are infected by the husband, the relatively high frequency of syphilis and, especially, of HSV-2 infection is not surprising. Although the presence of HSV-2 antibodies does not indicate the site or the route of infection, we may postulate that most infections were acquired through sexual contact and not through direct personal exposure. The prevalence of HSV-2 antibodies was very low among prepubertal children, unmarried women, and married women less than 20 years old. HSV-2 infection was associated with syphilis and indicators of STI. Considering that herpes is a lifelong infection, not cured by antimicrobial treatment, HSV-2 antibodies are a much more reliable indicator of risky behaviour than $T$ pallidum antibodies.

Screening of pregnant women for syphilis is claimed to be very cost effective, even if the prevalence is less than 1 in $10000 .{ }^{15}$ According to the diagnostic criteria used in the present study, $3 \%$ of the women had active syphilis, classified as latent syphilis of unknown duration, and $0.2 \%$ had an old infection as defined by a non-reactive RPR test combined with a positive TPHA/TPPA. The discrepancy between active and old infections would indicate a recent outbreak of syphilis. However, this is very unlikely. The age related increase of "active" syphilis, the absence of seroconversion among initially negative women, the high proportion of low RPR titres, the small proportion of women with $T$ pallidum $\operatorname{IgM}$ antibodies or showing a significant decline or rise of the RPR titre indicate that most women had not active disease. Nevertheless, the significant association with fetal death suggests strongly that syphilis is still a major health problem in Bangladesh, as in many other developing countries. ${ }^{16}$ Although HCV may be transmitted by sexual contact, preliminary data from female sex workers show that the prevalence of $\mathrm{HCV}$ infection is not different from women in the general population. In developing countries HBV infection is often acquired during childhood and is generally attributed to poor sanitation, malnutrition, and the use of contaminated blood or needles. Considering the high prevalence of $\mathrm{HBcAg}$ antibodies among all age groups as well as the lack of association with STI and their indicators, most HBV infections were probably not transmitted through sexual contact. Policy makers in Bangladesh believe that the very low prevalence rate of HIV infection is due to faith and rigid attitude towards premarital and extramarital sex. The present data on genital herpes suggest that the attitude towards "illegal" sex is not so rigid as is generally believed. HIV prevention programmes should focus not only the "high risk" groups such as sex workers and their clients, but also the general population.

This research was supported by the Belgian Administration for Development Cooperation and the ICDDR,B: Centre for Health and Population Research. The centre is supported by countries, donor agencies, and others who share its concern for the health and population problems of developing countries. The TPPA diagnostic kits were provided free of cost by The TPPA diagnostic kits were provided free of cost by
ameris Ltd, Antwerp, Belgium. Anowar Hossein, Clinical Lameris Ltd, Antwerp, Belgium. Anowar Hossein, Clinical
Laboratory ICDDR,B, provided serum from prepubertal girls. Contributors: JB contributed to the design, execution, analysis, Contributors: JB contributed to the design, execution, analysis,
nd interpretation of the study; JA, NA, and NB supervised the and interpretation of the study; JA, NA, and NB supervised the
nurse; MR and SN executed the laboratory tests in ICDDR; MVR and JV confirmed the laboratory findings.

1 Brunham RC, Embree JE. Sexually transmitted diseases: current and future dimensions of the problem in the third world. In: A Germain, KK Holmes, P Piot, JN Wasserheit, eds. Reproductive tract infections. Global impact and priorities for women's reproductive health. New York: Plenum Press, for women's reproductive health. New York: Plenum Press,

2 Hawkes S, Morison L, Foster S, et al. Reproductive-tract infections in women in low-income, low-prevalence situations: assessment of syndromic management in Matlab, Bangladesh. Lancet 1999;354:1776-81.

3 Nugent RP, Krohn MA, Hillier SL. Reliability of diagnosing bacterial vaginosis is improved by a standardized method of Gram stain interpretation. F Clin Microbiol 1991;29:297301

4 World Health Organization. Management of patients with sexually transmitted diseases. Report of a WHO Study Group. Geneva: WHO Technical Report Series, 810, 1991.

5 Sexually Transmitted Disases Treatment Guidelines. MMWR Recommendations and Reports. US Department of Health and Human Services. Public Health Service. Atlanta, GA: Centers for Disease Control and Prevention, 1993:42:RR-14

6 Bogaerts J, Ahmed J, Akhter N, et al. Sexually transmitted infections in a basic healthcare clinic in Dhaka, Bangladesh: syndromic management for cervicitis is not Bangladesh: syndromic management

7 Meda N, Sangaré, Lankoandé S, et al. Pattern of sexually transmitted diseases among pregnant women in Burkina Faso, West Africa: potential for a clinical management based on simple approaches. Genitourin Med 1997;73: 188-93 
8 Behets F, Desormeaux J, Joseph D, et al. Control of sexually transmitted disease in Haiti: results and implications of a baseline study among pregnant women living
shantytowns. F Infect Dis 1995;172:764-7.

9 Gertig DM, Kapiga SH, Shao JF, et al. Risk factors for sexually transmitted disease among women attending family planning clinics in Dar-es-Salaam, Tanzania. Genitourin Med 1997;73:39-43.

10 Mayaud P, Grosskurth H, Changalucha J, et al. Risk assessment and other screening options for gonorrhoea and chlamydial infections in women attending rural Tanzanian clinics. Bull World Health Organ 1995;73:621-30.

11 Thomas T, Choudhri S, Kariuki C, et al. Identifying cervical infection among pregnant women in Nairobi, Kenya: limitations of risk assessment and symptom-based approaches. Genitourin Med 1996;72:334-8.
12 Naved RT. RTI/STD and risky sexual behavior in a "conservative" society. Working paper. Save the Children (USA), Bangladesh Field Ofree, 1997.

13 Khan MA, Rahman M, Khanam PA, et al. Awareness of sexually transmitted disease among women and service providers in rural Bangladesh. Int $\mathcal{F}$ STD AIDS 1997;8: 688-96.

14 Caldwell B, Pieris I, Barkat-e-Kuda, et al. Sexual regimes and sexual networking: the risk of an HIV/AIDS epidemic in Bangladesh. Soc Sci Med 1999;48:1103-16.

15 Stray-Pedersen B. Economic evaluation of maternal screening to prevent congenital syphilis. Sex Transm Dis 1983;10: $167-72$.

16 Temmerman M, Lopita MI, Sanghvi HCG, et al. The role of maternal syphilis, gonorrhoea and HIV-1 infections in spontaneous abortion. Int f STD AIDS 1992;3:418-22. 